\section{Development and validation of a novel customized medium for the marine microbial culture}

\author{
Md. Morshedul Alam, ${ }^{\#}$, Redwan Ahmed\#, Md. Ariful Amin, Mohammad Nazir \\ Hossain \\ Department of Genetic Engineering \& Biotechnology, Marine Biotechnology Lab, \\ Bangladesh.Bangabandhu Sheikh Mujibur Rahman Maritime University, Mirpur-12, Dhaka-1216
}

Bioresearch Communications

Volume 7, Issue 2, July 2021

DOI:

https://doi.org/10.3329/brc.v7i2.54374

\begin{abstract}
Marine microbes are difficult to culture and hence it takes expensive measure to study. Mostly metagenomics are suggested for marine microbial characterization instead of culturing them in a suitable medium. In this study, a new approach has been developed to culture the marine microorganisms. We customized the LB agar and liquid medium by adjusting the $\mathrm{pH}$ and salt concentration at seawater level and then studied the microbial load, their growth rate by turbidity assay and also identified their morphology through gram staining. This study ensures the comfortable growth of marine microbes at the laboratory level, which would further help the marine biotechnologists and microbiologists to work smoothly with marine microbial samples.
\end{abstract}

KEYWORDS: Marine microbes, marine microbial growth medium, novel customized medium.
CORRESPONDING AUTHOR: Md. Morshedul Alam, Ph.D, Department of Genetic Engineering \& Biotechnology Marine Biotechnology Lab, Bangabandhu Sheikh Mujibur Rahman Maritime University, Mirpur-12, Dhaka-1216, Bangladesh. Email: rummanarahim@yahoo.com

\section{Introduction}

Ocean is vast reservoir of lots of resources for the mankind. It is also a big source of biodiversity. The most genetic diversity in the ocean is microbial, which resides in bacteria, archaea, protists, and viruses. In deed, by weight, the life in the ocean is mostly microbial (Hunter, 2011) ${ }^{1}$. Concerning the number, about $90 \%$ of biological life forms are viruses, $8-9 \%$ are prokaryotes and the rests are protists (Suttle, 2005, 2007, Whiteman et al, 1989) ${ }^{2-4}$. On an average, there are about 10 million viruses and 1 million prokaryotes in one milliliter of seawater sample, though it is variable based on the sampling location and ocean depth (Hennes and Suttle, 1995) ${ }^{5}$.

Now a day, the scope of the use of various useful genes available in marine microbes, due to the diversity and number of marine microbes for biotechnological applications are increasing exponentially. It prompts a paradigm shift in our knowledge of the ocean. Study of marine microbes is of great interest due to the presence of various genes with virtually unlimited potential for development and exploitation. Since, the importance of the study of marine microbes is increasing day by day, the next question comes in front that how to culture and store them. It is known that marine microbes are unculturable and, hence, it is a burning issue to make it unculturable to culturable one. Though in most cases scientists are dependent on metagenomics to study these marine microbes, but it is cost effective.

From the keywords that marine microbes are unculturable, a hypothesis has come in mind that for the marine microbial growth, providing ocean environment should be ensured. Keeping this in consideration, for the first time we propose a newly developed customized medium for the comfortable marine microbial growth. In this study we customize the LB medium to grow the marine microorganisms in the laboratory environment.

\section{Materials and Methods \\ Preparation of normal and customized LB Medium}

To make the normal LB medium, we used Bactotrypton, Yeast extract, $\mathrm{NaCl}$, Tris- $\mathrm{HCl}(\mathrm{pH}$ 7.5) as the medium composition. For the customized medium, we used normal LB (Bactotrypton, yeast extract, $\mathrm{NaCl}$, Tris- $\mathrm{HCl}$ ) agar medium and liquid medium with some modifications. To support the marine environment, we changed the salt $(\mathrm{NaCl})$ concentration to sea water level, roughly about $35 \mathrm{gm} / \mathrm{L}$, and also adjusted the $\mathrm{pH}$ level to 8.2. These modified mediums were used in this study.

\section{Sample collection}

Marine water samples were collected both from the Saint Martin's Island costal area and the various locations of Cox's Bazar costal areas of the Bay of Bengal, Bangladesh. Every sample was collected in pre-sterilized glass vials and stored for a short period of time at $-4^{\circ} \mathrm{C}$ temperature. The experiments were performed after three days of sample collection.

Culture of marine microbes in customized LB agar medium At first, we prepared customized LB agar medium for spread culture. To do so, we performed vortex mix the sample and spread about $120 \mu \mathrm{l}$ of marine sample directly to the medium, followed by air incubation at $37^{\circ} \mathrm{C}$ temperature. We incubated the marine microbes for 4-5 days and observed the culture growth status everyday. After the incubation period, microbial colonies were counted to know the microbial load in each sampling area. 


\section{Turbidity assay study}

For the turbidity assay, we used both sterile normal and customized liquid medium. Single bacterial colony was picked up using sterile toothpick into $3 \mathrm{ml}$ of liquid medium followed culturing in a shaking incubator at $37^{\circ} \mathrm{C}$ temperature for 3 days. After the incubation period, we took the absorbance at $\mathrm{OD}_{600}$ in a spectrophotometer.

\section{Gram staining}

Morphology of the isolated microbes was performed using well-established Gram staining procedure. In brief, after colony smearing and heat-fixing, the cells were flooded one by one with crystal violet, grams iodine, ethanol and safranin. After the completion of staining procedure, the cells were observed under light microscope at $40 \mathrm{X}$ magnification.

\section{Results}

Marine microbes can grow only in customized LB agar medium

To know whether our customized medium can support the marine microbial growth, first we spread our sample in customized LB agar plate and incubated. After 4-5 days incubation at $37^{\circ} \mathrm{C}$, we found substantial numbers of microbial colonies on customized LB agar plate (Fig. 1B). On the other hand, we didn't find any microbial colonies on the normal LB agar medium (Fig. 1A). This data suggests that the newly developed customized medium is compatible for marine microbial growth.

\section{Fig. 1}

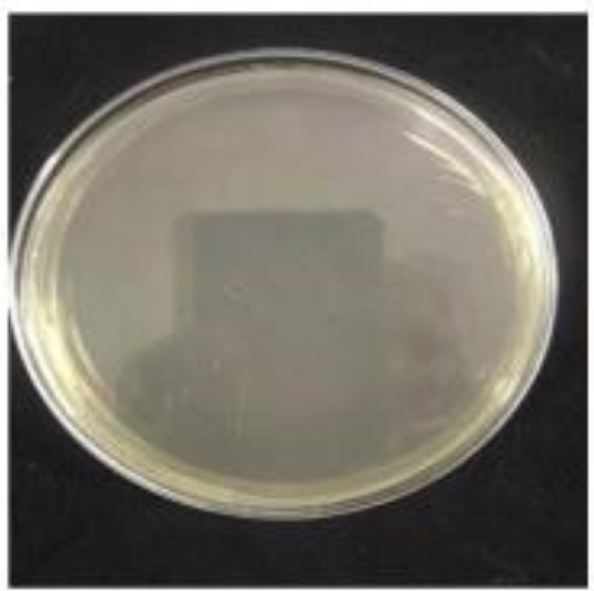

A.

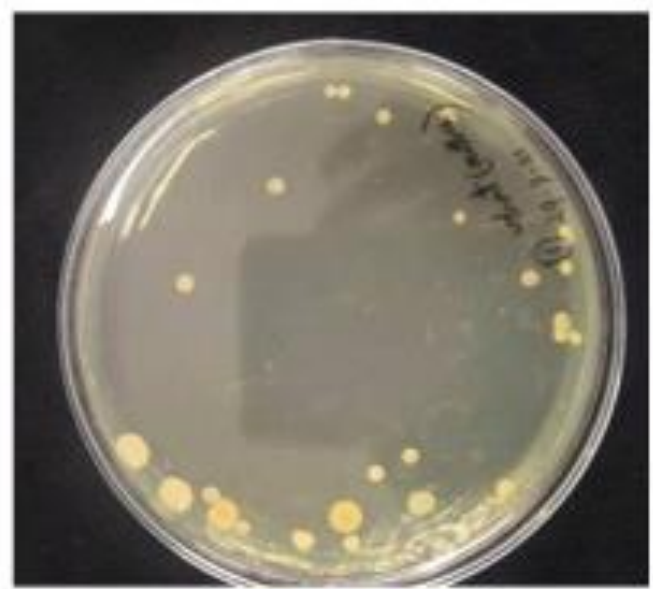

B.

Figure 1. Marine microbial cell growth on (A) normal LB agar medium and (B) customized LB agar medium. In both cases the minimum incubation time was 4 days to see the tiny colonies and 5 days to see bigger sized colony. The incubation temperature was $37^{\circ} \mathrm{C}$.

\section{Microbial load can be counted on customized medium with samples from distant marine areas}

To validate our newly developed medium, we collected marine samples from distant costal regions of Saint Martin's Island and Cox's Bazar. Culturing those samples on customized medium also allowed the growth of microbes at the similar incubation time period in the laboratory. We have counted the colony numbers of those samples to determine the microbial load at each costal site of the Bay of Bengal (Fig. 2A-D). This data suggests the validity of the newly developed customized LB medium for marine microbial growth. 
Fig. 2

A.

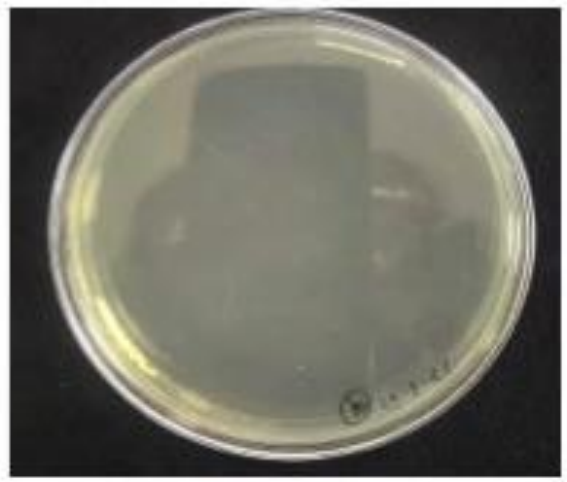

D.

\begin{tabular}{|c|c|}
\hline Sample ID & Cfu/L seawater \\
\hline CB 01 & $225 \pm 16 \times 10^{5}$ \\
\hline CB 02 & $24 \pm 13 \times 10^{5}$ \\
\hline CB 03 & $0.5 \pm 6 \times 10^{5}$ \\
\hline CB 04 & $1.0 \pm 5 \times 10^{5}$ \\
\hline CB 05 & $1.3 \pm 7 \times 10^{5}$ \\
\hline CB 06 & $27.9 \pm 11 \times 10^{5}$ \\
\hline CB 07 & $15.5 \pm 9 \times 10^{5}$ \\
\hline SM 01 & $2.0 \pm 8 \times 10^{5}$ \\
\hline
\end{tabular}

c.

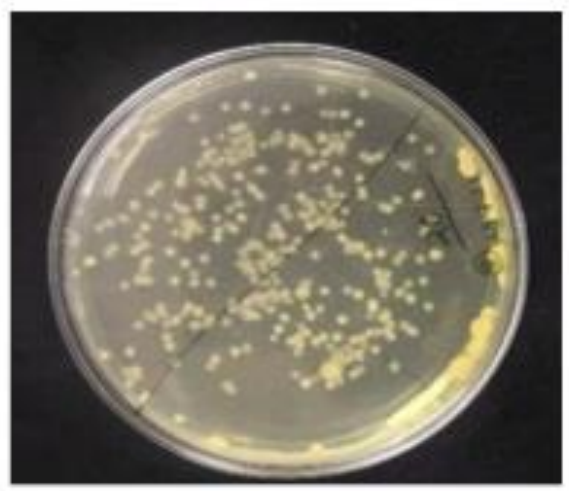

B.

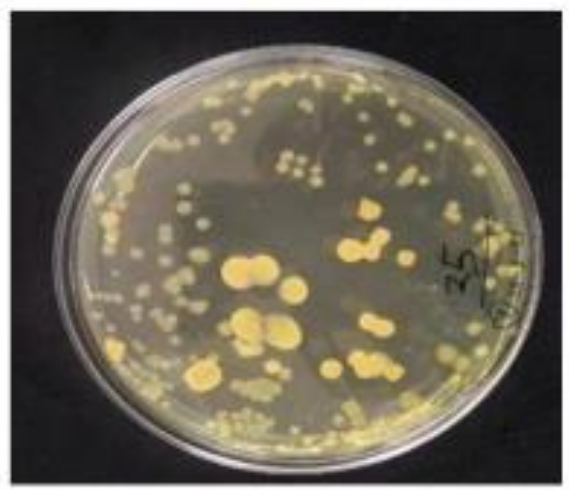

Figure 2. Marine microbial cell growth from distant costal region on customized medium. (A) Normal LB agar growth, (B-C) two representative customized LB agar plate with samples collected from two different locations of the costal region of Cox's Bazar. (D) Corresponding microbial colony numbers of various marine water samples from distant regions. Triplicate data were represented as $\mathrm{cfu} / \mathrm{L}$ seawater sample and mean \pm SD.

\section{Shaking incubation allows faster growth of marine microbes} in the customized medium

To decipher a suitable growth condition, we performed turbidity assay under shaking incubation for 3 days at $37^{\circ} \mathrm{C}$. Our data showed that microbial culture growth in customized medium was faster than culturing in customized LB agar medium as expected (Fig. 3A-D), which means that the incubation time is shorter ( 3 days) in shaking condition and the microbial growth is significant higher $(p<0.001)$ in the customized liquid culture medium than the normal liquid medium (Fig. 3E). Our data supports the notion that shaking culture takes shorter incubation time for marine microbial growth. 
Fig. 3

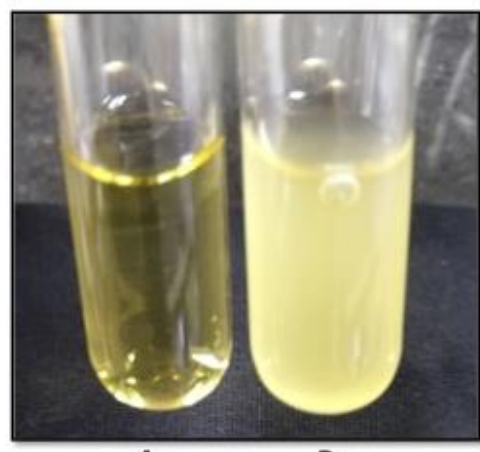

A.

B.
E.

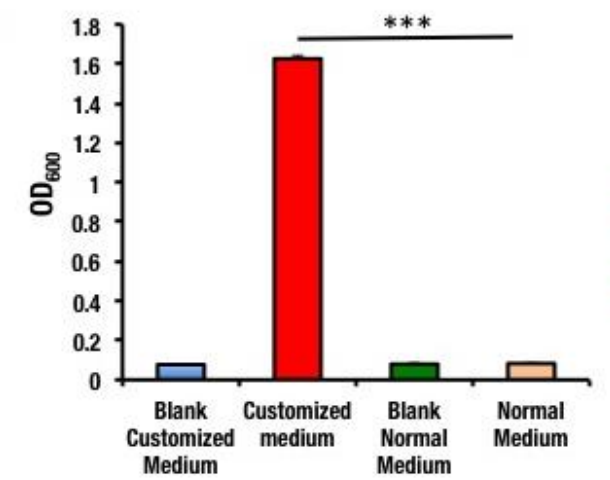

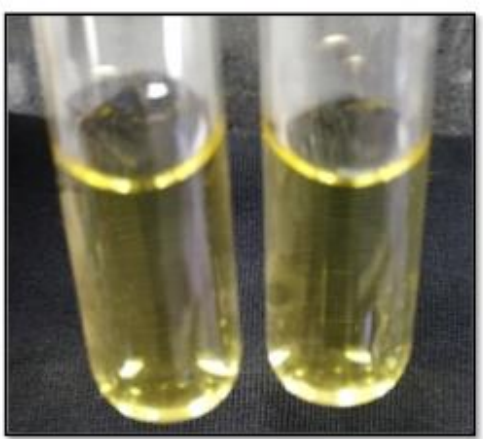

C.
D.
口Blank Customized Medium

aCustomized medium

口Blank Normal Medium

口Normal Medium

Figure 3. Marine microbial cell growth in liquid customized medium and normal medium. (A) customized medium without inoculation, (B) customized medium with marine microbial sample, (C) normal LB medium, (D) normal LB medium inoculated with marine microorganism. (E) growth rate study of marine microorganisms in customized medium and normal medium. Absorbance was taken at $\mathrm{OD}_{600}$ using spectrophotometer.

\section{Morphology of the marine microbes were detected}

To identify any microbe, it is a very common practice to observe their morphological structure. In this line we performed Gram staining using our collected marine water samples. We confirmed both gram positive (+ve) and gram negative (-ve) bacterial population in our isolated samples (Fig. 4A-B). It suggests that our newly developed customized medium would also support to conduct morphological structure study of marine microbial samples.

Fig. 4

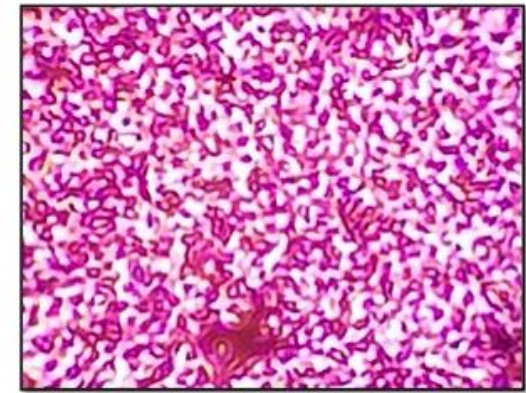

A.

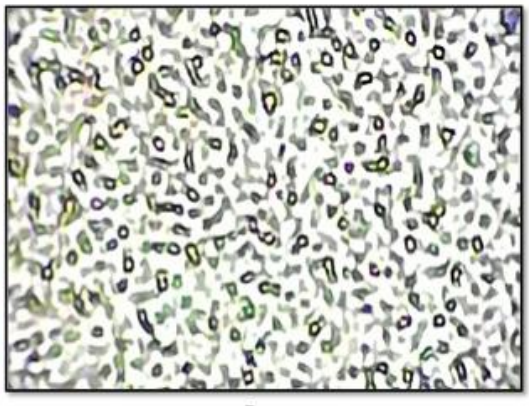

B.

Figure 4. Gram staining of marine microbial samples grown in customized LB medium. (A) gram negative organism isolated from Saint Martin's Island, (B) gram positive organism isolated from Cox's Bazar. Two representative data has been shown. 


\section{Discussion}

Culturing the uncultured is a big challenge specially, in line with the marine microbial samples. Mostly scientists depend on metagenomics strategy to study the marine microbial samples, as it is very difficult to culture the microbes in a suitable medium. So that developing new medium to culture marine microbial samples would make it easy to study and store them for further use. In this study we developed a new novel medium to culture those uncultured one. We modified the LB medium compositions and then used that medium to culture marine water samples to identify marine microbes. We successfully confirmed the microbial growth in our newly developed medium in both agar plate and liquid culture conditions and finally we identified the morphology of the culture.

In this study we modified the medium compositions of LB medium to ensure marine environment. For microbial growth they need nutrient sources as well as their supportive ecosystem. Keeping this in mind, we adjusted the medium $\mathrm{pH}$ into seawater level ( $\mathrm{pH}$ 8.2), so microbes would be able to satisfy their normal metabolic activities. Similarly, we also adjusted the salt concentration level, which was near about seawater level at which normal terrestrial microbes are completely unable to survive. Our customized medium very comfortably supported the marine microbial growth as it was observed by comparing with normal medium condition (Fig. 1-3). Shaking culture condition was also sharply differentiated the customized medium and normal medium (Fig. 3). This method would also help to identify the microbial culture at laboratory condition (Fig. 4). The growth rate of the marine microbes were a bit slow if we compare with normal terrestrial microbes in the LB agar medium, which generally takes 18 24hrs, but in our developed medium it took about 3-5 days. We customized only two vital factors, $\mathrm{pH}$ and salt concentration, we propose that with further modification, it will support more effective growth of marine microbes.

Though it was the very simple and first initiative to culture the marine microbes at laboratory condition, it would have some limitations as well. We cultured and detected only some extreme halophillic bacteria in our newly developed medium, but we are not sure about marine viruses or fungi culture in this medium, which require further study and we are now engaged in this research.

\section{Conclusion}

This is a very first study to grow the marine microbes in laboratory medium. Our newly developed customized medium allows marine microbes, which is uncultured into a cultured one. This method will help the marine microbiologists to study and culture marine microbial samples very easily. We have some limitations to study in a more scientific way and probably that would be the reason for slow growth rate. We are still improving our techniques to come out with a satisfactory level.

\section{Acknowledgement}

We are thankful to Dr. Ferdousi Begum (Assistant Professor), Ms. Farhana Akter (Lecturer) and Mr. Masud-ul-Alam (Lecturer), Bangabandhu Sheikh Mujibur Rahman Maritime University, Bangladesh for providing some samples as a kind gift from Cox's bazar location to conduct this experiment.

\section{Author Contribution}

M.M.A. conceived the idea, designed the research, performed and supervised the experiments, analyzed the data, wrote and edited the manuscript; R.A. performed the experiments, analyzed the data; M.A.A. performed experiments; M.N.H. analyzed the data.

\section{References}

1. Hunter, P. (2011), "Massing Life", EMBO Reports, 11(7):511-514.

2. Suttle, C.A. (2005), "Viruses in the sea", Nature 437:356361.

3. Suttle, C.A. (2007), "Marine viruses: Major players in the global ecosystem". National Review of Microbiology, 5(10):801-812.

4. Whiteman, W.B., Coleman D.C. and Wiebe, W.J. (1989), "Prokaryotes: The unseen majority". Proceedings of the National Academy of Sciences, 95(12):6578-6583.

5. Hennes, K.P. and Suttle, C.A. (1995), “ Direct counts of viruses in natural waters and laboratory cultures by epifluorescence microscopy". Limnology and Oceanography, 40:1050-1055. 